\title{
Cerebrospinal fluid levels of interleukin-8 in delirium, dementia and cognitively healthy
}

\section{patients.}

Muhammad Umar Sajjad ${ }^{1}$, Kaj Blennow ${ }^{2,3}$, Anne Brita Knapskog ${ }^{4}$, Ane-Victoria Idland ${ }^{1,5}$,

Farrukh Abbas Chaudhry ${ }^{1,8}$, Torgeir Bruun Wyller ${ }^{5}$, Henrik Zetterberg ${ }^{2,3,6,7}$, Leiv Otto Watne ${ }^{1,5}$

${ }^{1}$ Institute of Basic Medical Sciences, University of Oslo, Oslo, Norway

${ }^{2}$ Clinical Neurochemistry Laboratory, Sahlgrenska University Hospital, Mölndal, Sweden

${ }^{3}$ Department of Psychiatry and Neurochemistry, Institute of Neuroscience and Physiology, the Sahlgrenska Academy at the University of Gothenburg, Mölndal, Sweden

${ }^{4}$ Department of Geriatric Medicine, Oslo University Hospital, Oslo, Norway

${ }^{5}$ Oslo Delirium Research Group, Department of Geriatric Medicine, Oslo University Hospital,

Oslo, Norway

${ }^{6}$ Department of Neurodegenerative Disease, UCL Institute of Neurology, London, United Kingdom

${ }^{7}$ UK Dementia Research Institute at UCL, London, United Kingdom

${ }^{8}$ Department of Plastic and Reconstructive Surgery, Oslo University Hospital, Oslo, Norway

Running title: CSF IL-8 levels in delirium

Corresponding address: Muhammad Umar Sajjad, Institute of Basic Medical Sciences, Department of Molecular Medicine, University of Oslo, Postboks 1110, Blindern, 0317, OSLO, Norway. Telephone: + 47 93038867. E-mail: m.u.sajjad@medisin.uio.no 


\section{$\underline{\text { ABSTRACT }}$}

Delirium is a common and serious complication in geriatric patients. The pathophysiology of delirium is not known. The objective of the current study was to test the hypothesis that cerebrospinal fluid (CSF) levels of inflammatory markers at the time of spinal anesthesia for hip surgery are associated with delirium.

In total 133 hip fracture patients and 125 cognitively healthy controls undergoing elective surgery, together with 73 Alzheimer's disease (AD) dementia patients, were recruited at Oslo University Hospital and Diakonhjemmet Hospital, Oslo, Norway.

Delirium was evaluated daily in hip fracture patients by the Confusion Assessment Method (CAM). Depression was evaluated by Cornell Scale for Depression in Dementia (CSDD). Tumor necrosis factor alpha (TNF- $\alpha$ ), interleukin-1beta (IL-1 $\beta$ ) and interleukin-8 (IL-8) levels were measured in CSF using a Mesoscale Discovery (MSD) immunoassay.

Hip fracture patients had significantly higher IL-8 levels $(p<0.001)$ compared to cognitively healthy controls or patients with stable AD dementia. Furthermore, preoperative IL-8 levels were significantly higher $(p=0.013)$ in hip fracture patients who developed delirium (incident delirium) after surgery as compared to patients with no delirium. However, subgroup analyses showed that IL-8 levels were only significantly higher in delirium patients without dementia $(p=$ 0.006). In contrast, depression subgroup analysis showed that IL-8 concentration was significantly higher $(p=0.002)$ in delirium patients with depression. Both TNF- $\alpha$ and IL-1 $\beta$ were undetected in most patients. 
Our study suggests that IL-8 levels are associated with delirium onset and that underlying depression or dementia influences IL-8 levels.

KEYWORDS: Delirium, Cytokines, Alzheimer's disease, Depression

List of Abbreviations: Alzheimer's disease (AD); Tumor Necrosis Factor alpha (TNF- $\alpha$ ); Interleukin-1beta (IL-1 $\beta$ ); Interleukin-8 (IL-8); Cerebrospinal Fluid (CSF); Confusion Assessment Method (CAM); Cornell Scale for Depression in Dementia (CSDD); Subsyndromal Delirium (SSD); Amyloid- $\beta$ Precursor Protein (A $\beta P P)$; Microtubule-associated protein tau (tau); phospho-tau (p-tau); Lower Detection Limit (LDL) 


\section{INTRODUCTION}

Delirium is an acute neuropsychiatric syndrome characterized by fluctuating awareness, inattention and cognitive impairment [1]. A significant proportion (25-50\%) of hospitalized elderly patients develop delirium $[2,3]$. It is particularly common among elderly patients experiencing traumatic injury such as hip fracture [4] and is associated with increased mortality and morbidity [5].

Pre-existing dementia is a strong risk factor for delirium [6]. On the other hand, it is also increasingly evident that delirium is a risk factor for dementia [7]. Furthermore, several studies have reported that depression is a risk factor for delirium and depression is common among elderly admitted to the hospital $[8,9]$. There might be overlapping features and common pathological mechanisms between delirium and dementia, such as impaired stress and inflammatory responses, altered monoamine neurotransmission and melatonergic activity $[9,10]$.

Pathophysiology of delirium remains unknown although many pathways have been suggested [11, 12]. Neuroinflammation is one of the most studied areas and is associated with delirium onset [13, 14]. Several studies have reported elevated levels of pro-inflammatory cytokines in the serum and CSF of delirium patients [15-21]. Neuroinflammation plays a vital role in the brain vulnerability for long-term structural and functional consequences and neuropathological changes such as microglial activation and production of pro-inflammatory cytokines accelerate upon additional systemic inflammatory response due to injury or infection [22]. These findings have been reproduced in the preclinical animal models where systemic inflammation has been reported to accelerate the existing neuropathology such as microglial priming and acute cognitive and motor deficit in ME7 models of prion disease [23, 24]. 
Several attempts have been made to understand the role of inflammation by studying inflammatory markers and pro-inflammatory cytokines such as TNF- $\alpha$, interleukin (IL)-1 $\beta$, IL-6 and IL-8 in relation to delirium onset in hip fracture patients. Elevated blood levels of inflammatory markers and pro-inflammatory cytokines including TNF- $\alpha$, IL-1 $1 \beta$, IL-6 and IL- 8 of hip fracture patients that developed delirium have been reported [16, 18, 19, 25]. One study reported higher CSF IL-8 levels in delirium patients in a small cohort of hip fracture patients [26], while another study in which CSF cytokines levels were examined in patients with or without postoperative delirium and found no significant change in pro-inflammatory cytokines including IL-8 [27]. In fact, this study suggested that delirium onset is due to dysfunctional neuroinflammatory response that result in reduced anti-inflammatory cytokines rather than an increase in pro-inflammatory cytokines.

The role of pro-inflammatory cytokines and chemokines has also been extensively studied in cellular and animal models of AD. These studies have reported that pro-inflammatory cytokines and chemokines are associated with altered amyloid- $\beta$ precursor protein $(\mathrm{A} \beta \mathrm{PP})$ processing that result in increased $\mathrm{A} \beta$ production, protein aggregation and hyperphosphorylation of microtubuleassociated protein tau (p-tau) [28]. In addition to this, levels of several pro-inflammatory cytokines/chemokines have been reported to be elevated in the blood and CSF of AD patients, although, these finding are fairly contradicting [29]. Moreover, AD associated polymorphisms of TNF- $\alpha$, IL-1 $\beta$, IL-8 among other cytokines have also been identified [30, 31].

One the other hand, the role of cytokines and chemokines are not so well explored in the CSF of depressed geriatric patients. One study suggested elevated CSF levels of IL-6 and IL-8 in geriatric female patients with current depression [32]. In the contrary, Stubner et al., 1999 reported decreased CSF IL-6 and soluble IL-6 receptor levels in depressed geriatric patients [33]. Moreover, elevated levels of pro-inflammatory cytokines such as IL- $1 \beta$ and IL- 6 have been reported in the 
CSF of non-geriatric patients with depression disorders [34-36], although, some contradictory findings have also been reported [37, 38].

In summary, CSF pro-inflammatory cytokines levels in hip fracture patients with delirium have been reported with contradictory data $[26,39]$. Therefore, we aimed to elucidate the role of proinflammatory cytokines such as TNF- $\alpha$, IL- $1 \beta$ and IL- 8 in CSF in delirium in a larger cohort and with additional control groups such as cognitively healthy controls and patients with stable AD.

\section{MATERIAL AND METHODS}

CSF was collected from patients admitted to Oslo University Hospital and Diakonhjemmet Hospital, Oslo Norway.

\section{HIP FRACTURE PATIENTS}

Hip fracture patients were recruited from the Oslo Orthogeriatric Trial (inclusion 2009 - 2012), a randomized controlled trial evaluating the effect of orthogeriatric care on cognitive function [4042]. Participants with CSF available $(n=137)$ were included in the current study. Patients were assessed daily for delirium, preoperatively and until the fifth postoperative day, by the Confusion Assessment Method (CAM), which is a diagnostic algorithm consisting of four items: 1) acute onset and fluctuation course, 2) inattention, 3) disorganized thinking, and 4) altered level of consciousness. The diagnosis of delirium requires the presence of item 1 and 2 and either 3 or 4 . [43]. The CAM scores were based on a 10-30 minutes interview with the patient, in combination with information from nurses, close relatives and hospital records reporting symptoms during the last 24 hours. Patients were regularly assessed on weekdays only, but staff members that had been

working during weekends were interviewed every Monday and the case notes were scrutinized in 
order to reveal potential episodes of delirium. Delirium was categorized into different subcategories such as preoperative, incident (patients who were free from delirium when CSF was taken, but developed delirium later), and subsyndromal (SSD, defined as at least two positive CAM features [pre- or postoperatively] but never fulfilled criteria for full delirium). SSD patients were included as a part of no delirium group in the analyses unless otherwise stated. Each participant's fulfillment of ICD-10 criteria for dementia was evaluated by two consultants (one geriatrician and one geriatric psychiatrist) prior to the fracture, based on all existing data (except delirium status during admission), as described previously [40]. Cornell Scale for Depression in Dementia (CSDD) was used to assess symptoms of depression and score of 8 or more was considered the cutoff of depression [44]. Delirium status of three hip fracture patients were missing and therefore excluded from the analyses.

\section{COGNITIVELY HEALTHY CONTROL GROUP}

We recruited 172 participants ( $\geq 65$ years) in the COGNORM-study during 2012 and 2013 at Oslo University Hospital and Diakonhjemmet Hospital, Oslo, as described previously [45]. These participants were undergoing elective gynecological, orthopedic or urological surgery in spinal anesthesia. The participant's cognitive functions were tested by using a multi-domain battery of cognitive tests before surgery and at yearly follow up controls. Patients without CSF samples $(n=13)$, suspected undiagnosed dementia any time within the first 5 years of follow up $(n=15)$ or Mini Mental State Examination (MMSE) score <28 at baseline $(\mathrm{n}=19)$ were excluded from this study. 


\section{STABLE AD DEMENTIA PATIENTS}

The $73 \mathrm{AD}$ dementia patients were included at the memory clinic at Oslo University Hospital between 2009 and 2014. They all went through a comprehensive examination according to a standardized research protocol including cognitive assessment (among others MMSE, the clock drawing tests and the trail making test A and B), and physical examination with blood sampling, lumbar puncture, and magnetic resonance imaging (MRI). The patients were diagnosed according to the National Institute on Aging-Alzheimer's Association (NIA-AA) criteria for Alzheimer's disease [46]. Depressive symptoms were assessed with the CSDD as stated earlier.

\section{CSF SAMPLING AND HANDLING}

CSF was collected in polypropylene tubes before administration of the anesthetic agent in all patients undergoing surgery and as part of the diagnostic work up for the AD patients. CSF samples were centrifuged, aliquoted and stored at $-80^{\circ}$ C. Samples were analyses at the Clinical Neurochemistry Laboratory at Sahlgrenska University Hospital (Mölndal, Sweden). CSF concentrations of IL-8, TNF- $\alpha$ and IL-1 $\beta$ was analyzed using a Mesoscale Discovery (MSD) immunoassay (V-PLEX Human Proinflammatory Panel I), following the recommendations by the manufacturer.

\section{STANDARD PROTOCOL APPROVAL, REGISTRATION AND PATIENT CONSENTS}

The study was conducted in accordance with the Declaration of Helsinki. The data and CSF samples were collected after informed consent from the patient and/or proxy (if patients were unable to consent due to cognitive impairment), as approved by the Regional Committee for Medical and Health Research Ethics (REK 2009/450, 2011/2052, and 2017/371). 


\section{$\underline{\text { STATISTICS }}$}

SPSS version 25 was used for statistical analysis and figures were drawn in GraphPad prism version 7.04. Data were not normally distributed and therefore non-parametric tests were performed. Mann-Whitney $U$ test and Kruskal-Wallis test were used for continuous variable. Chisquare and Fisher's exact test were used for categorical variables. Both dementia and depression are risk factors for delirium and therefore we conducted subgroup analyses on dementia or depression status. Since SSD has been reported to be clinically closer to delirium as compared to no delirium, we analyzed our data with and without SSD as a part of the no delirium group [47]. CSF IL-8 concentrations were natural log-transformed for linear regression analysis.

\section{$\underline{\text { RESULTS }}$}

IL-8, IL-1 $\beta$ and TNF- $\alpha(\mathrm{pg} / \mathrm{mL})$ were measured in the CSF of 134 hip fracture patients, 125 elective surgery patients (cognitively healthy controls) and 73 patients with stable AD dementia (table 1). IL-1 $\beta$ and TNF- $\alpha$ could be detected in less than $10 \%$ of the patients (table 1 ). Therefore, we only proceeded with IL-8 concentration of different patient groups for further analysis. IL-8 was detected in all samples except from one patient in the hip fracture group. This patient was excluded from all analyses. Hip fracture patients were significantly older (median (IQR) 84 (7989) years) compared to the cognitively healthy (71 (68-76.5) years) and AD dementia (66 (6070.5 ) years) group (both $\mathrm{p}<0.001$ ) and more hip fracture patients were female ( $73 \%$ vs $50 \%$ vs $58 \%$, see table 1 for details). 


\section{CSF IL-8 IN HIP FRACTURE PATIENTS}

Overall, there was a trend towards higher CSF IL-8 levels in hip fracture patients with incident delirium (118.1 (79.7-183.3) pg/mL) and preoperative delirium (99.5 (72.8-133.4) pg/mL) compared to those without delirium (85.8 (62.7-130.1) $\mathrm{pg} / \mathrm{mL}, p=0.057)$ (figure 1a). When SSD patients were excluded, IL-8 was significantly higher in incident delirium compared to no delirium (118.1 (79.7-183.3) pg/mL vs $78.6(59.7-127.4) \mathrm{pg} / \mathrm{mL}, \mathrm{p}=0.013$, figure $1 \mathrm{~b})$ and the association was upheld when adjusted for age (table 2).

In order to understand the role of underlying dementia pathology and CSF IL-8 concentration, we performed subgroup analyses of hip fracture patients according to delirium and dementia status. The CSF IL-8 concentration was significantly higher in delirium when compared to no delirium in patients without dementia (129.8 (91.8-172.8) $\mathrm{pg} / \mathrm{ml}$ vs $85.8(60.6-131.6) \mathrm{pg} / \mathrm{ml}, p=0.006)$ and the association was upheld when adjusted for age (table 2). In patients with dementia, there was no difference in IL-8 between those with and without delirium (89.6 (68.3-125.3) pg/ml vs $86.8(74.3-135.1) \mathrm{pg} / \mathrm{ml}, p=0.851)$.

Since depression has been associated with pro-inflammatory cytokines, we also run analyses on delirium subgroups according to depression status. We found that in patients with depression $(\mathrm{n}=$ 21) IL-8 was higher in delirium compared to those without delirium (92.9 (67.9-134.5) pg/ml vs $50.4(42.1-69.2) \mathrm{pg} / \mathrm{ml}, p=0.002)$. However, the association did not hold up when adjusted for age, possibly due to lack of statistical power (table 2). On the other hand, in patients without depression, $(n=100)$ there was no significant difference in IL-8 concentration between patients with and without delirium (99.6 (72.2-140.4) $\mathrm{pg} / \mathrm{ml}$ vs $92.9(69.7-136.8) \mathrm{pg} / \mathrm{ml}, p=0.565)$. 


\section{DEMENTIA}

In order to further explore the interplay between dementia and delirium on CSF IL-8 levels, we included two additional control groups; patients with stable AD dementia and cognitively healthy controls (table 1). Both AD dementia patients (42.1 (36.2-50.2) pg/mL) and cognitively healthy controls (46.9 (40.1-54.4) pg/mL) had significantly lower levels of CSF IL-8 concentration when compared to hip fracture group $(91.8(69.6-136.3) \mathrm{pg} / \mathrm{mL}, \mathrm{p}<0.001$ for both groups, table 1).

\section{CSF IL-8 AND AGE}

Since there is a significant difference in age between different patient groups (table 1), we conducted a spearman rank correlation analysis between age and IL-8 concentration within each patient group. There was no significant correlation between IL-8 concentration and age in any of the patients groups (supplementary table 1).

\section{$\underline{\text { DISCUSSION }}$}

To our knowledge this is the first study that compares CSF IL-8 levels in delirium patients, cognitively healthy controls and AD dementia patients side by side. Our data supports the neuroinflammatory hypotheses in delirium. However, in most samples we could not detect TNF$\alpha$ and IL- $\beta$. This could be due to sensitivity of the assays for both IL- $1 \beta$ and TNF- $\alpha$, although similar findings were reported previously in hip fracture patients [26].

There is some evidence in the literature that supports the role of pro-inflammatory cytokines in the pathophysiology of delirium, especially in studies examining cytokine levels in blood 
samples. However, there is an ambiguity with pro-inflammatory cytokines levels, including IL-8, in the CSF of hip fracture patients.

We found that IL- 8 concentration seemed to be impacted by delirium status at the time of sampling, with the highest levels in those about to develop delirium after surgery (incident delirium). It is well-established that IL-8 is an acute pro-inflammatory marker $[48,49]$ and has also been shown to be elevated in the CSF of multiple sclerosis (MS) [50], AD and Parkinson's disease (PD) patients as compared to age matched controls [51]. Highest IL-8 levels in incident delirium suggest a role of an acute inflammatory response in phase prior to a clinically evident delirium and this increase in CSF IL-8 concentration is not associated with age. In addition to this, underlying conditions such as dementia increase the vulnerability for preoperative delirium whereas non-demented patients are more likely to get incident delirium [52], and that suggests the involvement of different inflammatory pathways associated with delirium subtypes. Thus, these observations are relevant for our study.

IL-8 concentration was significantly higher in incident delirium only when SSD patients were excluded (figure 1b). It has been suggested that SSD falls between no delirium and delirium [53]. Recently a study by Yamada and colleagues further supports this idea where they showed that $33.9 \%$ of critically ill patient in an ICU setting had SSD and 9.5\% of these patients later developed delirium [54]. Our data supports that SSD is clinically relevant. Dementia subgroup analyses revealed that the observed differences in IL-8 in delirium were confined to patients free from pre-fracture dementia (figure 2a). This observation is consistent with an earlier study that showed decreased IL-8 CSF and serum levels in patients with AD. They also showed that IL-1 $\beta$ levels negatively correlates with MMSE score and suggested that pro-inflammatory markers such as IL-1 $\beta$ and IL-8 may have separate pathogenic mechanisms involved in AD [55]. Moreover, IL- 
8 levels have also been reported to be elevated in CSF samples of AD patients $[51,56,57]$ and this contradiction in the literature may well be due to the difference in sensitivity and robustness of the assay used and/or difference in patient cohorts in terms of pathology. Although, there was no significant difference observed in IL-8 levels between dementia and dementia free hip fracture patients (supplementary table 2). In order to further explore the role of IL-8 in delirium and dementia, we compared CSF IL-8 levels of cognitively healthy controls or stable AD patients with hip fracture group. Our analysis showed that patients with stable AD or cognitively healthy controls had significantly lower levels of IL-8 as compared to hip fracture patients (table 1). IL-8 has been shown to be elevated in both acute and chronic inflammatory diseases and we postulate that IL-8 levels are acutely elevated in hip fracture patient as compared to AD dementia patients and cognitively healthy controls. This could be due to acute nature of the fracture that as a result recruited immune cells particularly neutrophils, lymphocytes and monocytes responsible for producing pro-inflammatory cytokines including IL-8. It would be interesting to know how IL-8 levels are regulated in chronic neuroinflammatory condition like $\mathrm{AD}$ as compared to more acute scenario such as hip-fracture. However, this was not possible in this study since we didn't have samples from hip-fracture patients at different intervals after surgery.

To our knowledge, the role of pro-inflammatory cytokines in delirium and depression has never been studied together in the CSF of hip fracture patients. We showed that in hip fracture patients with depression, patients with delirium had significantly higher IL-8 levels as compared to those without delirium. An earlier study reported higher CSF IL-6 and IL-8 levels in geriatric female patients with current depression [32]. This observation suggest that IL-8 levels are regulated by underlying depression pathology and the difference in IL-8 level may be influenced by underlying depression and/or dementia state in a more complex fashion in hip fracture patients. 
Strengths of this study are the use of fairly large cohort of hip fracture patients with additional control groups that allowed us to understand the role of IL-8 and delirium in a broader context. Also, all of the analyses were conducted simultaneously and this allowed us to compare different groups side by side in order to understand the interplay between delirium, dementia and depression in hip fracture patients. We have conducted subgroup analyses since there is a significant overlap between delirium, dementia and depression. We anticipated that the relationship between IL-8, delirium, dementia and depression are crucial for cellular and molecular mechanisms for normal brain functions. It is important to interpret the data carefully and further experiments may be required for more thorough understanding of IL-8 in complex pathological scenario. A limitation is that we did not have access to follow-up samples during and after the procedure. CSF levels of IL-8 are known to increase dramatically hours after initiation of non-neurological surgery $[58,59]$, and it would have been highly interesting to examine this change in relation to delirium incidence. Unfortunately, we were unable to detect the TNF- $\alpha$ and IL-1 $\beta$ levels in the majority of the samples. Hence, there is a need to develop more sensitive and robust assays to analyze these two markers in delirium patients CSF samples.

In summary, our study showed that preoperative IL-8 was higher in hip fracture patients that developed delirium after surgery. Both preoperative dementia status and depression status seemed to influence the association between IL-8 and delirium, suggesting that the association between IL-8 levels and delirium are dependent on underlying pathology. 


\section{ACKNOWLEDGEMENTS}

We would like to thank the patients and staff at the Orthopedic Department, the Gynecology Department, the Urology Department, and the Anesthesiology Department at Oslo University Hospital and the Surgery Department and the Anesthesiology Department at Diakonhjemmet Hospital in Oslo. We also thank research nurses Elisabeth Fragaat and Tone Fredriksen for help in data collection and the laboratory technicians at the Clinical Neurochemistry Laboratory, Sahlgrenska University Hospital, for skillful technical analyses. The study was funded by the Norwegian Health Association, the South-Eastern Norway Regional Health Authorities and the Medical Student Research Program in Norway. The sponsors had no role in the design and conduct of the study, the collection, management, analysis, and interpretation of the data, or the preparation, review, and approval of the manuscript.

\section{CONFLICT OF INTEREST}

L.O.Watne reports no conflicts of interest. KB has served as a consultant or at advisory boards for Alector, Alzheon, CogRx, Biogen, Lilly, Novartis and Roche Diagnostics, and is a co-founder of Brain Biomarker Solutions in Gothenburg AB, a GU Ventures-based platform company at the University of Gothenburg, all unrelated to the work presented in this paper. HZ has served at scientific advisory boards for Roche Diagnostics, Wave, Samumed and CogRx, and is a cofounder of Brain Biomarker Solutions in Gothenburg AB, a GU Ventures-based platform company at the University of Gothenburg, all unrelated to the work presented in this paper.

HZ is a Wallenberg Academy Fellow supported by grants from the Swedish Research Council (\#2018-02532), the European Research Council (\#681712), Swedish State Support for Clinical Research (\#ALFGBG-720931) and the Olav Thon Foundation. 


\section{$\underline{\text { REFERENCES }}$}

[1] Davis DH, Barnes LE, Stephan BC, MacLullich AM, Meagher D, Copeland J, Matthews FE, Brayne C, Function MRCC, Ageing S (2014) The descriptive epidemiology of delirium symptoms in a large population-based cohort study: results from the Medical Research Council Cognitive Function and Ageing Study (MRC CFAS). BMC Geriatr 14, 87.

[2] Inouye SK, Westendorp RG, Saczynski JS (2014) Delirium in elderly people. Lancet 383, 911922.

[3] Jackson TA, Gladman JR, Harwood RH, MacLullich AM, Sampson EL, Sheehan B, Davis DH (2017) Challenges and opportunities in understanding dementia and delirium in the acute hospital. PLoS Med 14, e1002247.

[4] Watne LO, Idland AV, Fekkes D, Raeder J, Frihagen F, Ranhoff AH, Chaudhry FA, Engedal K, Wyller TB, Hassel B (2016) Increased CSF levels of aromatic amino acids in hip fracture patients with delirium suggests higher monoaminergic activity. BMC Geriatr 16, 149.

[5] Witlox J, Eurelings LS, de Jonghe JF, Kalisvaart KJ, Eikelenboom P, van Gool WA (2010) Delirium in elderly patients and the risk of postdischarge mortality, institutionalization, and dementia: a meta-analysis. JAMA 304, 443-451.

[6] Fong TG, Davis D, Growdon ME, Albuquerque A, Inouye SK (2015) The interface between delirium and dementia in elderly adults. Lancet Neurol 14, 823-832.

[7] Davis DH, Muniz-Terrera G, Keage HA, Stephan BC, Fleming J, Ince PG, Matthews FE, Cunningham C, Ely EW, MacLullich AM, Brayne C, Epidemiological Clinicopathological Studies in Europe Collaborative M (2017) Association of Delirium With Cognitive Decline in Late Life: A Neuropathologic Study of 3 Population-Based Cohort Studies. JAMA Psychiatry 74, 244-251.

[8] Cepoiu M, McCusker J, Cole MG, Sewitch M, Ciampi A (2007) Recognition of depression in older medical inpatients. J Gen Intern Med 22, 559-564.

[9] O'Sullivan R, Inouye SK, Meagher D (2014) Delirium and depression: inter-relationship and clinical overlap in elderly people. Lancet Psychiatry 1, 303-311.

[10] Miller AH, Maletic V, Raison CL (2009) Inflammation and its discontents: the role of cytokines in the pathophysiology of major depression. Biol Psychiatry 65, 732-741.

[11] Hshieh TT, Inouye SK, Oh ES (2018) Delirium in the Elderly. Psychiatr Clin North Am 41, 1-17.

[12] Mulkey MA, Hardin SR, Olson DM, Munro CL (2018) Pathophysiology Review: Seven Neurotransmitters Associated With Delirium. Clin Nurse Spec 32, 195-211.

[13] Cortese GP, Burger C (2017) Neuroinflammatory challenges compromise neuronal function in the aging brain: Postoperative cognitive delirium and Alzheimer's disease. Behav Brain Res 322, 269279.

[14] Simone MJ, Tan ZS (2011) The role of inflammation in the pathogenesis of delirium and dementia in older adults: a review. CNS Neurosci Ther 17, 506-513.

[15] Capri M, Yani SL, Chattat R, Fortuna D, Bucci L, Lanzarini C, Morsiani C, Catena F, Ansaloni L, Adversi M, Melotti MR, Di Nino G, Franceschi C (2014) Pre-Operative, High-IL-6 Blood Level is a Risk Factor of Post-Operative Delirium Onset in Old Patients. Front Endocrinol (Lausanne) 5, 173.

[16] de Rooij SE, van Munster BC, Korevaar JC, Levi M (2007) Cytokines and acute phase response in delirium. $J$ Psychosom Res 62, 521-525.

[17] van den Boogaard M, Kox M, Quinn KL, van Achterberg T, van der Hoeven JG, Schoonhoven L, Pickkers P (2011) Biomarkers associated with delirium in critically ill patients and their relation with long-term subjective cognitive dysfunction; indications for different pathways governing delirium in inflamed and noninflamed patients. Crit Care 15, R297. 
[18] van Munster BC, Bisschop PH, Zwinderman AH, Korevaar JC, Endert E, Wiersinga WJ, van Oosten HE, Goslings JC, de Rooij SE (2010) Cortisol, interleukins and S100B in delirium in the elderly. Brain Cogn 74, 18-23.

[19] van Munster BC, Korevaar JC, Zwinderman AH, Levi M, Wiersinga WJ, De Rooij SE (2008) Time-course of cytokines during delirium in elderly patients with hip fractures. J Am Geriatr Soc 56, 1704-1709.

[20] Neerland BE, Hall RJ, Seljeflot I, Frihagen F, MacLullich AM, Raeder J, Wyller TB, Watne LO (2016) Associations Between Delirium and Preoperative Cerebrospinal Fluid C-Reactive Protein, Interleukin-6, and Interleukin-6 Receptor in Individuals with Acute Hip Fracture. J Am Geriatr Soc 64, 1456-1463.

[21] Hall RJ, Watne LO, Cunningham E, Zetterberg H, Shenkin SD, Wyller TB, MacLullich AMJ (2017) CSF biomarkers in delirium: a systematic review. Int J Geriatr Psychiatry.

[22] Holmes C (2013) Review: systemic inflammation and Alzheimer's disease. Neuropathol Appl Neurobiol 39, 51-68.

[23] Combrinck MI, Perry VH, Cunningham C (2002) Peripheral infection evokes exaggerated sickness behaviour in pre-clinical murine prion disease. Neuroscience 112, 7-11.

[24] Cunningham C, Campion S, Lunnon K, Murray CL, Woods JF, Deacon RM, Rawlins JN, Perry VH (2009) Systemic inflammation induces acute behavioral and cognitive changes and accelerates neurodegenerative disease. Biol Psychiatry 65, 304-312.

[25] Beloosesky Y, Hendel D, Weiss A, Hershkovitz A, Grinblat J, Pirotsky A, Barak V (2007) Cytokines and C-reactive protein production in hip-fracture-operated elderly patients. J Gerontol A Biol Sci Med Sci 62, 420-426.

[26] MacLullich AM, Edelshain BT, Hall RJ, de Vries A, Howie SE, Pearson A, Middleton SD, Gillies F, Armstrong IR, White TO, Cunningham C, de Rooij SE, van Munster BC (2011) Cerebrospinal fluid interleukin-8 levels are higher in people with hip fracture with perioperative delirium than in controls. J Am Geriatr Soc 59, 1151-1153.

[27] Westhoff D, Witlox J, Koenderman L, Kalisvaart KJ, de Jonghe JF, van Stijn MF, Houdijk AP, Hoogland IC, Maclullich AM, van Westerloo DJ, van de Beek D, Eikelenboom P, van Gool WA (2013) Preoperative cerebrospinal fluid cytokine levels and the risk of postoperative delirium in elderly hip fracture patients. J Neuroinflammation 10, 122.

[28] Domingues C, da Cruz ESOAB, Henriques AG (2017) Impact of Cytokines and Chemokines on Alzheimer's Disease Neuropathological Hallmarks. Curr Alzheimer Res 14, 870-882.

[29] Brosseron F, Krauthausen M, Kummer M, Heneka MT (2014) Body fluid cytokine levels in mild cognitive impairment and Alzheimer's disease: a comparative overview. Mol Neurobiol 50, 534544.

[30] Qin B, Li L, Wang S, Wu J, Huang Y, Zhou P, Bai J, Zheng Y (2016) Interleukin-8 gene polymorphism -251T>A contributes to Alzheimer's disease susceptibility. Medicine (Baltimore) 95, e5039.

[31] Su F, Bai F, Zhang Z (2016) Inflammatory Cytokines and Alzheimer's Disease: A Review from the Perspective of Genetic Polymorphisms. Neurosci Bull 32, 469-480.

[32] Kern S, Skoog I, Borjesson-Hanson A, Blennow K, Zetterberg H, Ostling S, Kern J, Gudmundsson P, Marlow T, Rosengren L, Waern M (2014) Higher CSF interleukin-6 and CSF interleukin-8 in current depression in older women. Results from a population-based sample. Brain Behav Immun 41, 55-58.

[33] Stubner S, Schon T, Padberg F, Teipel SJ, Schwarz MJ, Haslinger A, Buch K, Dukoff R, Lasser R, Muller N, Sunderland T, Rapoport SI, Moller HJ, Hampel H (1999) Interleukin-6 and the soluble IL-6 receptor are decreased in cerebrospinal fluid of geriatric patients with major depression: no alteration of soluble gp130. Neurosci Lett 259, 145-148.

[34] Sasayama D, Hattori K, Wakabayashi C, Teraishi T, Hori H, Ota M, Yoshida S, Arima K, Higuchi T, Amano N, Kunugi H (2013) Increased cerebrospinal fluid interleukin-6 levels in 
patients with schizophrenia and those with major depressive disorder. $J$ Psychiatr Res 47, 401406.

[35] Levine J, Barak Y, Chengappa KN, Rapoport A, Rebey M, Barak V (1999) Cerebrospinal cytokine levels in patients with acute depression. Neuropsychobiology 40, 171-176.

[36] Lindqvist D, Janelidze S, Hagell P, Erhardt S, Samuelsson M, Minthon L, Hansson O, Bjorkqvist M, Traskman-Bendz L, Brundin L (2009) Interleukin-6 is elevated in the cerebrospinal fluid of suicide attempters and related to symptom severity. Biol Psychiatry 66, 287-292.

[37] Hestad KA, Engedal K, Whist JE, Aukrust P, Farup PG, Mollnes TE, Ueland T (2016) Patients with depression display cytokine levels in serum and cerebrospinal fluid similar to patients with diffuse neurological symptoms without a defined diagnosis. Neuropsychiatr Dis Treat 12, 817822.

[38] Carpenter LL, Heninger GR, Malison RT, Tyrka AR, Price LH (2004) Cerebrospinal fluid interleukin (IL)-6 in unipolar major depression. J Affect Disord 79, 285-289.

[39] Westhoff D, Witlox J, van Aalst C, Scholtens RM, de Rooij SE, van Munster BC, de Jonghe JF, Houdijk AP, Eikelenboom P, van Westerloo DJ, van de Beek D, van Gool WA, Koenderman L (2015) Preoperative protein profiles in cerebrospinal fluid in elderly hip fracture patients at risk for delirium: A proteomics and validation study. BBA Clin 4, 115-122.

[40] Watne LO, Torbergsen AC, Conroy S, Engedal K, Frihagen F, Hjorthaug GA, Juliebo V, Raeder J, Saltvedt I, Skovlund E, Wyller TB (2014) The effect of a pre- and postoperative orthogeriatric service on cognitive function in patients with hip fracture: randomized controlled trial (Oslo Orthogeriatric Trial). BMC Med 12, 63.

[41] Watne LO, Hall RJ, Molden E, Raeder J, Frihagen F, MacLullich AM, Juliebo V, Nyman A, Meagher D, Wyller TB (2014) Anticholinergic activity in cerebrospinal fluid and serum in individuals with hip fracture with and without delirium. J Am Geriatr Soc 62, 94-102.

[42] Wyller TB, Watne LO, Torbergsen A, Engedal K, Frihagen F, Juliebo V, Saltvedt I, Skovlund E, Raeder J, Conroy S (2012) The effect of a pre- and post-operative orthogeriatric service on cognitive function in patients with hip fracture. The protocol of the Oslo Orthogeriatrics Trial. BMC Geriatr 12, 36.

[43] Inouye SK, van Dyck CH, Alessi CA, Balkin S, Siegal AP, Horwitz RI (1990) Clarifying confusion: the confusion assessment method. A new method for detection of delirium. Ann Intern Med 113, 941-948.

[44] Barca ML, Eldholm RS, Persson K, Bjorklof GH, Borza T, Telenius E, Knapskog AB, Braekhus A, Saltvedt I, Selbaek G, Engedal K (2018) Cortisol levels among older people with and without depression and dementia. Int Psychogeriatr, 1-5.

[45] Idland AV, Sala-Llonch R, Borza T, Watne LO, Wyller TB, Braekhus A, Zetterberg H, Blennow K, Walhovd KB, Fjell AM (2016) CSF neurofilament light levels predict hippocampal atrophy in cognitively healthy older adults. Neurobiol Aging 49, 138-144.

[46] McKhann GM, Knopman DS, Chertkow H, Hyman BT, Jack CR, Jr., Kawas CH, Klunk WE, Koroshetz WJ, Manly JJ, Mayeux R, Mohs RC, Morris JC, Rossor MN, Scheltens P, Carrillo MC, Thies B, Weintraub S, Phelps CH (2011) The diagnosis of dementia due to Alzheimer's disease: recommendations from the National Institute on Aging-Alzheimer's Association workgroups on diagnostic guidelines for Alzheimer's disease. Alzheimers Dement 7, 263-269.

[47] Boettger S, Nunez DG, Meyer R, Richter A, Schubert M, Jenewein J (2018) Subsyndromal delirium in the intensive care setting: Phenomenological characteristics and discrimination of subsyndromal delirium versus no and full-syndromal delirium. Palliat Support Care 16, 3-13.

[48] Hirao Y, Kanda T, Aso Y, Mitsuhashi M, Kobayashi I (2000) Interleukin-8 - An early marker for bacterial infection. Laboratory Medicine 31, 39-44.

[49] Volpin G, Cohen M, Assaf M, Meir T, Katz R, Pollack S (2014) Cytokine levels (IL-4, IL-6, IL-8 and TGFbeta) as potential biomarkers of systemic inflammatory response in trauma patients. Int Orthop 38, 1303-1309. 
[50] Matejcikova Z, Mares J, Sladkova V, Svrcinova T, Vyslouzilova J, Zapletalova J, Kanovsky P (2017) Cerebrospinal fluid and serum levels of interleukin-8 in patients with multiple sclerosis and its correlation with Q-albumin. Mult Scler Relat Disord 14, 12-15.

[51] Zhang J, Sokal I, Peskind ER, Quinn JF, Jankovic J, Kenney C, Chung KA, Millard SP, Nutt JG, Montine TJ (2008) CSF multianalyte profile distinguishes Alzheimer and Parkinson diseases. Am J Clin Pathol 129, 526-529.

[52] McCusker J, Cole M, Dendukuri N, Han L, Belzile E (2003) The course of delirium in older medical inpatients: a prospective study. J Gen Intern Med 18, 696-704.

[53] Cole M, McCusker J, Dendukuri N, Han L (2003) The prognostic significance of subsyndromal delirium in elderly medical inpatients. J Am Geriatr Soc 51, 754-760.

[54] Yamada C, Iwawaki Y, Harada K, Fukui M, Morimoto M, Yamanaka R (2018) Frequency and risk factors for subsyndromal delirium in an intensive care unit. Intensive Crit Care Nurs 47, 1522.

[55] Hesse R, Wahler A, Gummert P, Kirschmer S, Otto M, Tumani H, Lewerenz J, Schnack C, von Arnim CA (2016) Decreased IL-8 levels in CSF and serum of AD patients and negative correlation of MMSE and IL-1beta. BMC Neurol 16, 185.

[56] Hu WT, Howell JC, Ozturk T, Gangishetti U, Kollhoff AL, Hatcher-Martin JM, Anderson AM, Tyor WR (2019) CSF Cytokines in Aging, Multiple Sclerosis, and Dementia. Front Immunol 10, 480.

[57] Taipa R, das Neves SP, Sousa AL, Fernandes J, Pinto C, Correia AP, Santos E, Pinto PS, Carneiro P, Costa P, Santos D, Alonso I, Palha J, Marques F, Cavaco S, Sousa N (2019) Proinflammatory and anti-inflammatory cytokines in the CSF of patients with Alzheimer's disease and their correlation with cognitive decline. Neurobiol Aging 76, 125-132.

[58] Bromander S, Anckarsater R, Kristiansson M, Blennow K, Zetterberg H, Anckarsater H, Wass CE (2012) Changes in serum and cerebrospinal fluid cytokines in response to non-neurological surgery: an observational study. J Neuroinflammation 9, 242.

[59] Reinsfelt B, Ricksten SE, Zetterberg H, Blennow K, Freden-Lindqvist J, Westerlind A (2012) Cerebrospinal fluid markers of brain injury, inflammation, and blood-brain barrier dysfunction in cardiac surgery. Ann Thorac Surg 94, 549-555. 
Table 1: Background characteristics and CSF inflammatory markers comparison between hip fracture patients, cognitively healthy controls and Alzheimer's disease (AD) dementia patients

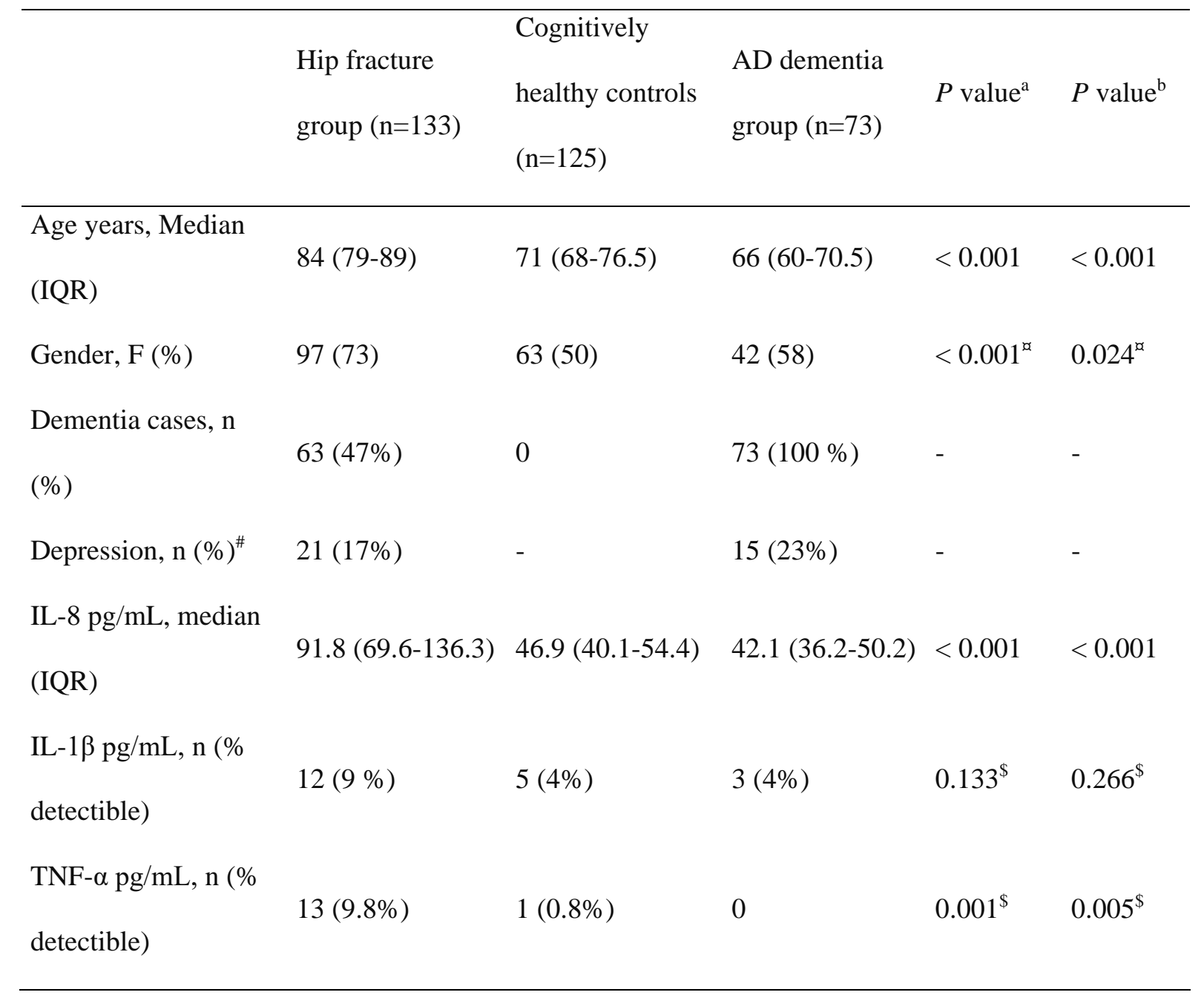

${ }^{\text {a }}$ Comparison between cognitively healthy and hip fracture patients

${ }^{\mathrm{b}}$ Comparison between hip fracture patients and $\mathrm{AD}$ patients

${ }^{\ltimes}$ Chi-Square $\mathrm{p}$ value was reported for both genders

\$Fisher's Exact p value was reported 
${ }^{\#}$ Depression defined as $\geq 8$ on Cornell scale in hip fracture patients and AD dementia patients. Data on depression was missing for 12 patients in hip fracture group and 8 patients in the AD dementia group. Mann-Whitney U test was perform to compare two different groups 
Table 2: Regression analysis of CSF IL-8 concentration (dependent variable) as a function of age and delirium (independent variables). CSF IL-8 values were transformed with the natural logarithm for approximation of residuals to Gaussian distribution.

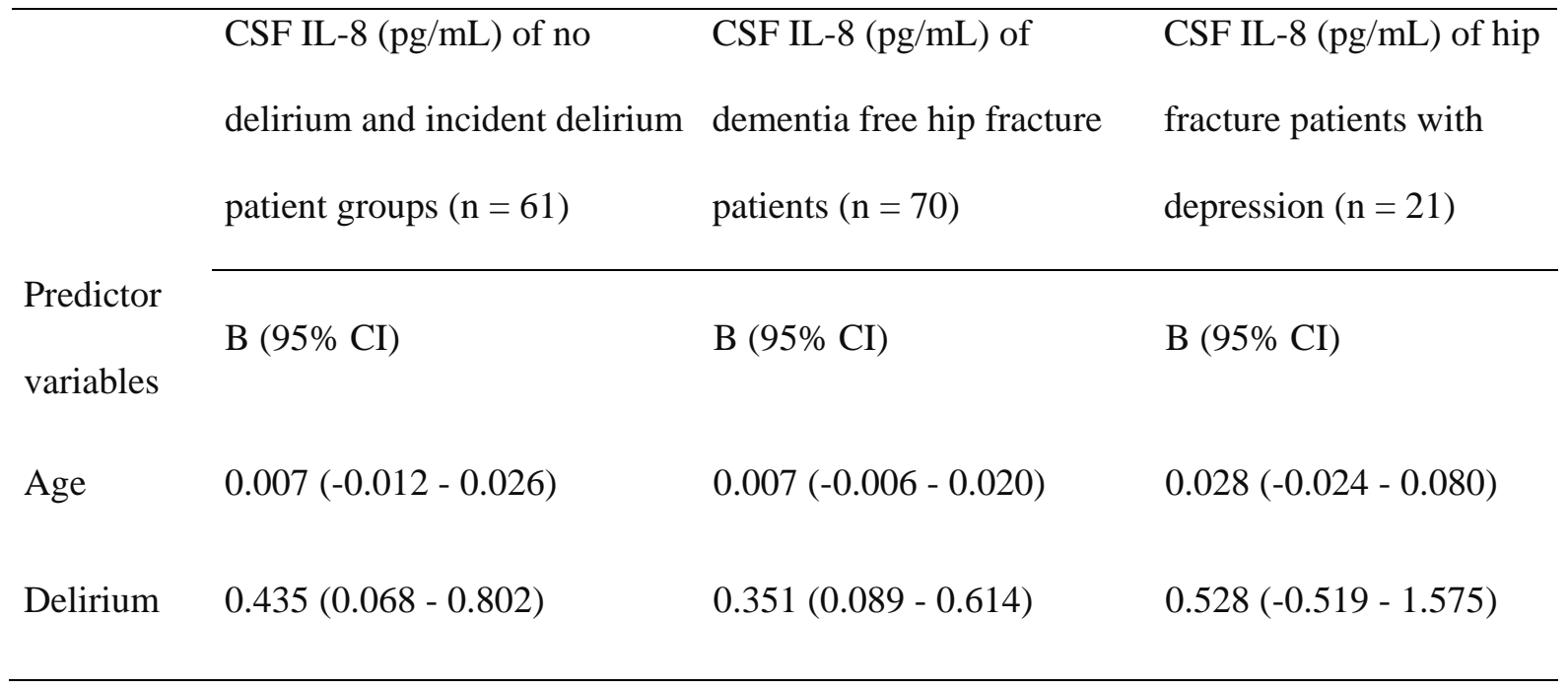


A With SSD

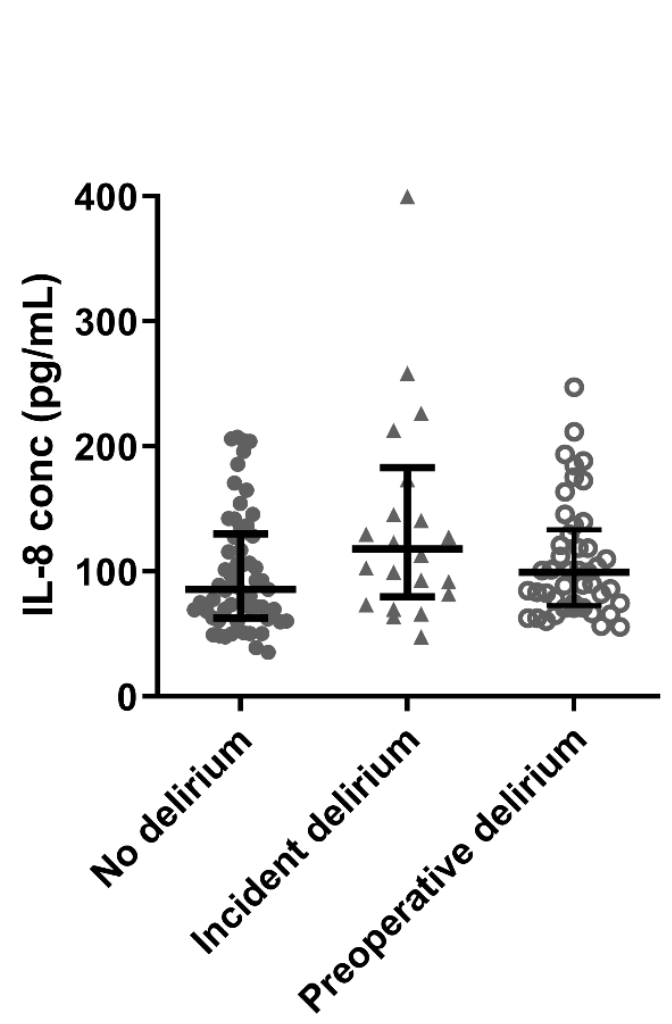

B Without SSD

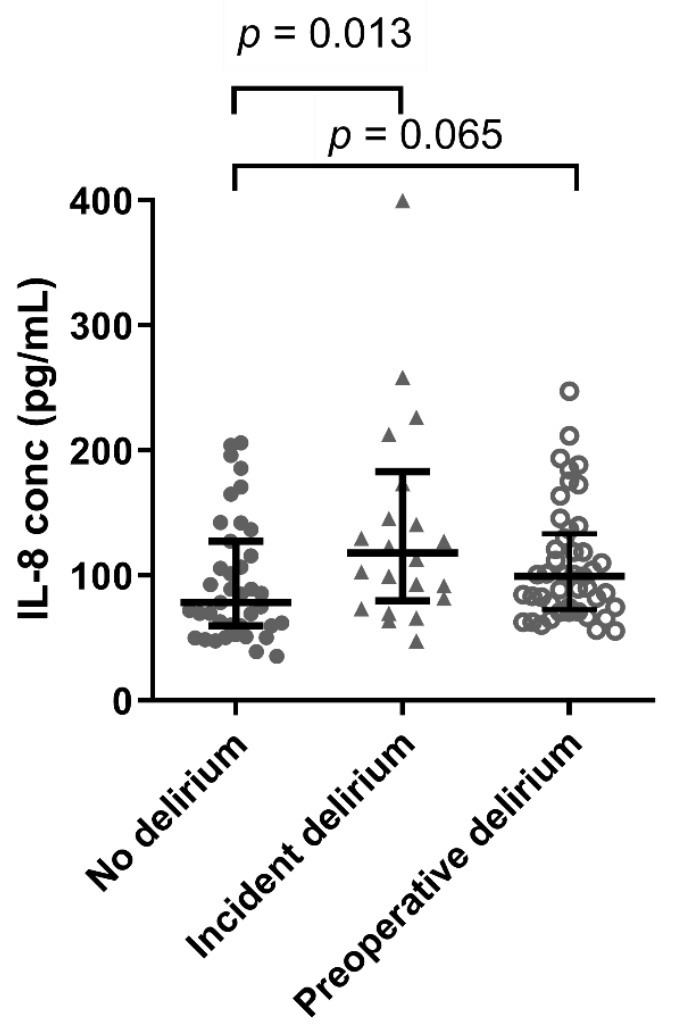

Figure 1: IL-8 concentrations in the CSF of hip fracture patients according to delirium subgroups.

SSD - Subsyndromal delirium 


\section{A: Without Dementia}

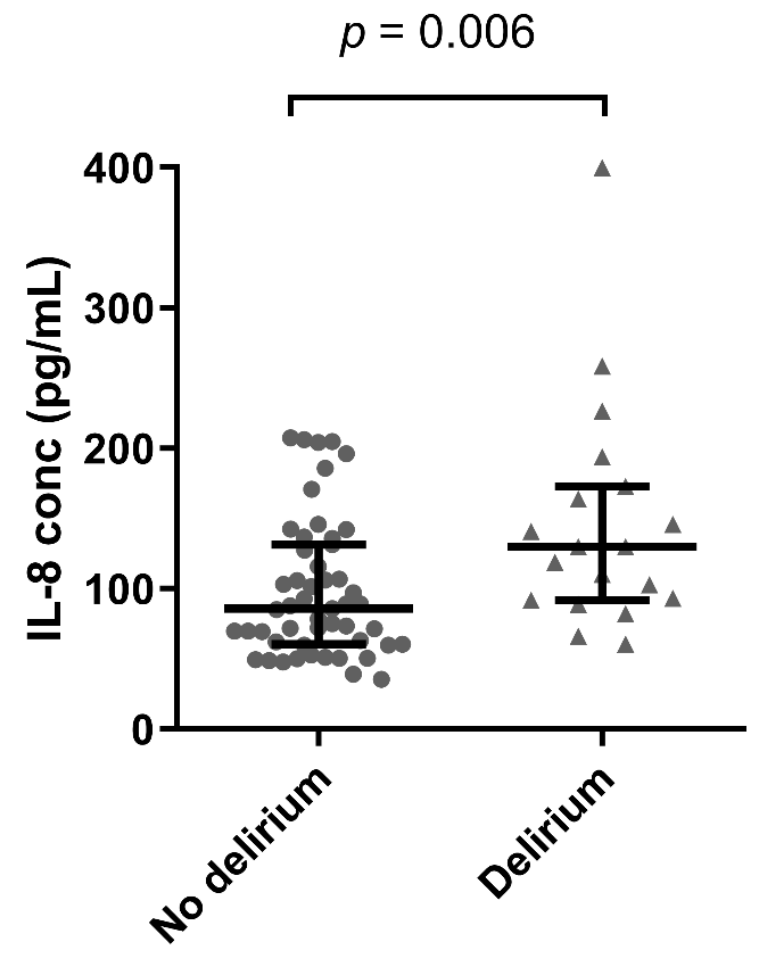

B: Dementia

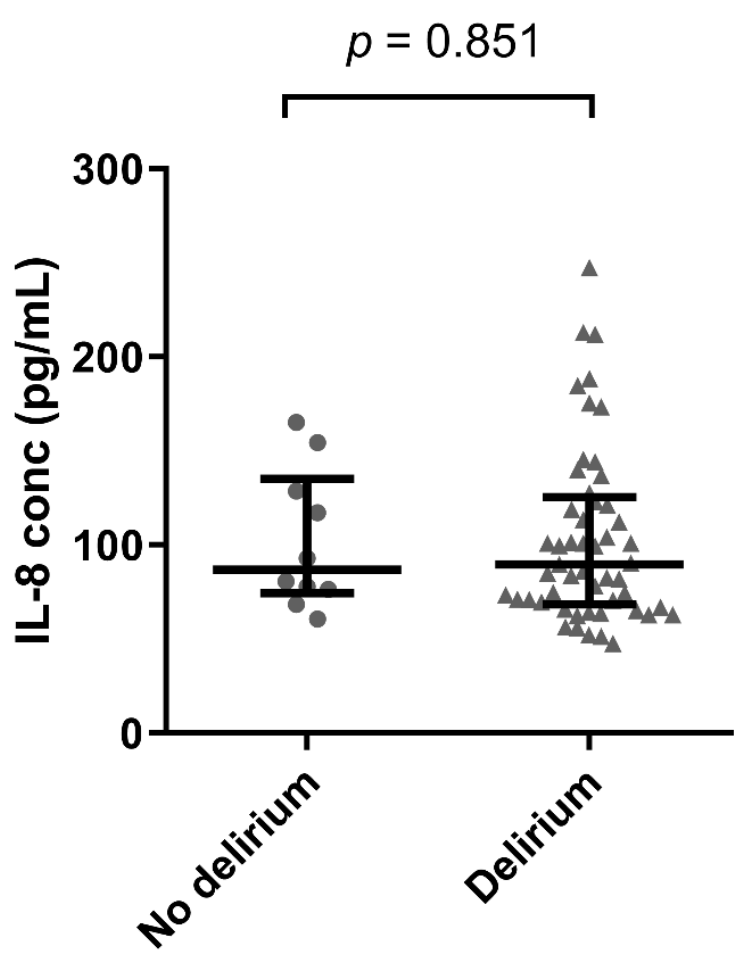

Figure 2: IL-8 concentration in the CSF of hip fracture patients according to delirium and dementia subgroups. 


\section{A: No Depression}

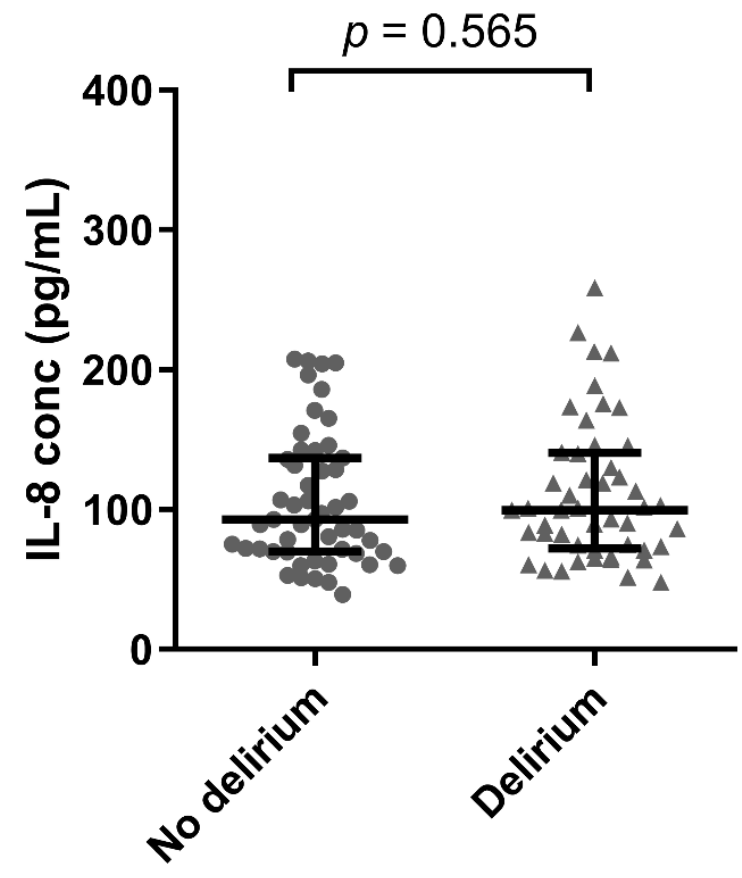

B: Depression

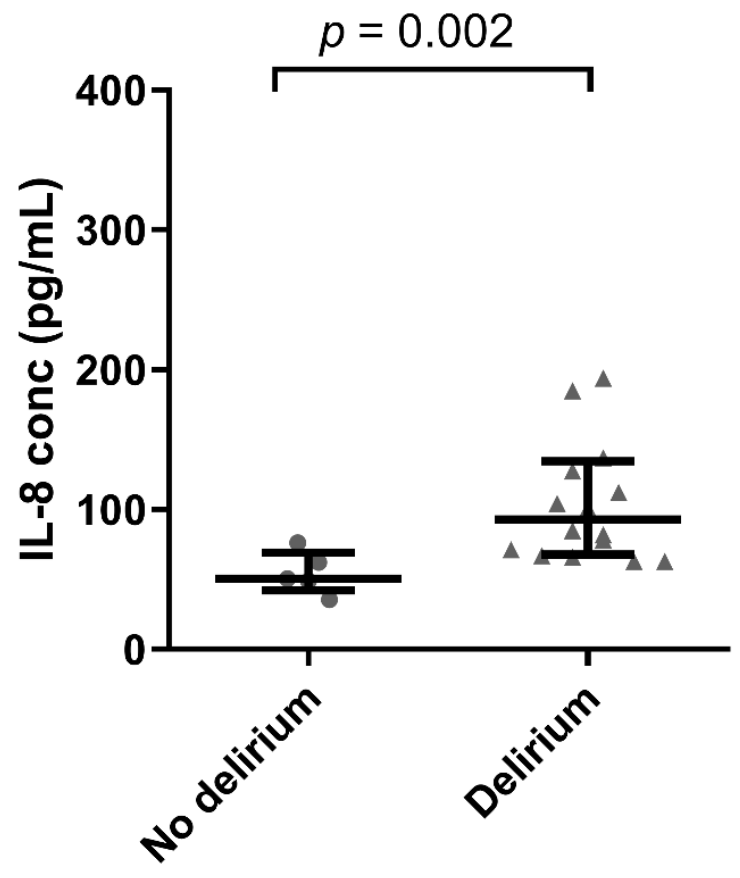

Figure 3: IL-8 concentration in the CSF of hip fracture patients according to delirium and depression subgroups. 


\section{Figure Captions and Legends}

Figure 1: IL-8 concentration in the CSF of hip fracture patients according to delirium subgroups. A) IL-8 concentration by delirium subgroups where the subsyndromal delirium (SSD) subgroup is part of the no delirium group $(n=61$, no delirium; $\mathrm{n}=22$, incident delirium; $\mathrm{n}=45$, preoperative delirium). Kruskal-Wallis test did not show significant difference between different groups ( $p=$ 0.057). One outlier $(y=3131)$ in the incident delirium had a value above the $y$-axis of this figure and was removed for visual purposes but not from the statistical analysis. B) IL-8 concentration by delirium subgroups without the SSD subgroup $(n=39$, no delirium; $n=22$, incident delirium; $\mathrm{n}=$ 45, preoperative delirium). Kruskal-Wallis test showed significant difference between different groups $(p=0.023)$. Mann-Whitney $\mathrm{U}$ test was conducted for comparison between no delirium and incident delirium $(p=0.013)$ or no delirium and preoperative delirium $(p=0.065)$. Delirium subcategories data was not available for five patients.

Figure 2: IL-8 concentration in the CSF of hip fracture patients according to delirium status and dementia subgroups. A) IL-8 concentration by delirium status in dementia free patients ( $n=51$, no delirium; $n=19$, delirium group). Mann-Whitney $\mathrm{U}$ test showed significant difference between no delirium and delirium groups $(p=0.006)$. B) IL-8 concentration by delirium status in dementia patients ( $n=10$, no delirium; $n=53$, delirium group). Mann-Whitney $\mathrm{U}$ test was conducted for statistical analysis and there was no significant difference between no delirium and delirium groups $(p=0.851)$. One outlier $(y=3131)$ in the delirium group had a value above the $y$-axis of this figure and was removed for visual purposes but not from the statistical analysis.

Figure 3: IL-8 concentration in the CSF of hip fracture patients according to delirium status and depression subgroups. A) IL-8 concentration in hip fracture patients by delirium status without 
depression ( $n=51$, no delirium; $n=49$, delirium; $p=0.565$ ). B) IL-8 concentration in hip fracture patients by delirium status with depression ( $n=5$, no delirium; $n=16$, delirium; Exact $p=0.002$ ). One outlier $(y=3131)$ in the delirium group had a value above the $y$-axis of this figure and was removed for visual purposes but not from the statistical analysis. Depression data was not available for 12 patients ( $n=5$, no delirium; $n=7$, delirium patients). Mann-Whitney $\mathrm{U}$ test was conducted for comparison between no delirium and delirium group. 
Supplementary Table 1: Correlations between CSF IL-8 and age in different patient groups

\begin{tabular}{lccc}
\hline Patient groups & $\mathbf{r}_{\mathbf{s}}$ & $\mathbf{p}$-value & $\mathbf{n}$ \\
\hline Cognitively healthy controls & 0.143 & 0.113 & 125 \\
Hip fracture group & 0.113 & 0.195 & 133 \\
AD dementia patients & 0.015 & 0.899 & 73 \\
\hline
\end{tabular}

Supplementary table 2: Comparison between dementia and dementia free patients in hip-fracture group

\section{Hip fracture patients}

\begin{tabular}{cccc} 
& No - dementia $(\mathbf{n}=\mathbf{7 0})$ & Dementia $(\mathbf{n}=\mathbf{6 3})$ & p-value \\
\hline Age years, Median (IQR) & $84(76-88)$ & $85(80-90)$ & $0.029^{*}$ \\
Gender, F (\%) & $51(73 \%)$ & $46(73 \%)$ & $0.984^{\#}$ \\
Depression, n (\%) & $5(8 \%)$ & $16(27 \%)$ & $0.006^{\#}$ \\
IL-8 pg/mL, median (IQR) & $93(68.6-141.2)$ & $89.6(69.7-127.5)$ & $0.815^{*}$ \\
\hline
\end{tabular}

*Mann-Whitney $U$ test was performed.

a) Data on depression was missing for 4 patients in the dementia group and 8 patients in the nodementia group.

${ }^{\#}$ Chi-Square $p$-values were reported. 